\title{
Development of Transport and Manipulation Robotic System for Operation on the Spacecraft Surface
}

\author{
I.Y. Dalyaev ${ }^{1}$, E.Y. Smirnova ${ }^{1}$, I.A. Vasiliev ${ }^{1}$, A.A. Koshurina ${ }^{2}$ and M.S. Krasheninnikov ${ }^{2}$ \\ ${ }^{1}$ 194064, Russia, St. Petersburg, Tihoreckij prospect, 21, CNII RTK \\ ${ }^{2} 603950$, Russia, Nizhny Novgorod, Minina street, 24, NNSTU named after R.E. Alekseev
}

\begin{abstract}
This work is devoted to creation of transport and manipulation robotic system (TMS) to work on the surface of the spacecraft. Undoubtedly, the TMS is a powerful tool in solving problems of the study of space, so it is evident that the role of space robotics is continuously increasing due to the need to develop and maintain global communications, navigation and surveillance, permanent manned and visited space stations and bases on the Moon and on the planets of the solar system. As a basic model of a transport subsystem we proposed the symmetrical structure with seven degrees of freedom. The paper describes the structure of the system and provides its kinematics. The article presents the data obtained from the simulation in the MSC.ADAMS package. Thanks to the simulation were obtained predictive characteristics and defined functions of the robotic system. This allowed us to define a technical way to create transport and manipulation robotic system to service the outer surface of the spacecraft. Also this paper deals with the grouped operation of interacting objects (manipulators). These methods of grouped operation are mainly used to control groups of mobile robots, but as specified in [1.2], the general principles of control of the group interaction do not depend on the physical nature (and moreover on the kinematic characteristics) of the interacting objects.
\end{abstract}

Keywords-transport and manipulation systems; robotics; group interaction; grippers; spacecraft; space

\section{INTRODUCTION}

Modern space robotics is a promising scientific and technical field, covering a comprehensive design, development, production and operation of robotic tools for space purposes. [3] Such systems are being actively developed and used in international space projects of the US, Canada, Japan, the European Union and Russia. Modern robotic systems differ in size, structure, purpose, methods of work and control.

Nowadays there are manipultion systems for space applications that can be used to perform a strictly limited list of technological operations. The limited range of operations is connected with fixed fastening of manipulation systems on the outer surface of the spacecraft (SC), with the complexity of their control during the EVA (extravehicular activity).

The experience of existing of the manipulation systems on the ISS shows that their capacity is not sufficient to carry out all necessary maintenance work spectrum. In some cases, the astronauts have to go into space and perform a series of complex operations manually [4].

\section{MODEL}

As a basic model of kinematics of the transport subsystem we propose the use of symmetrical structure with seven degrees of freedom, shown in Figure 1.

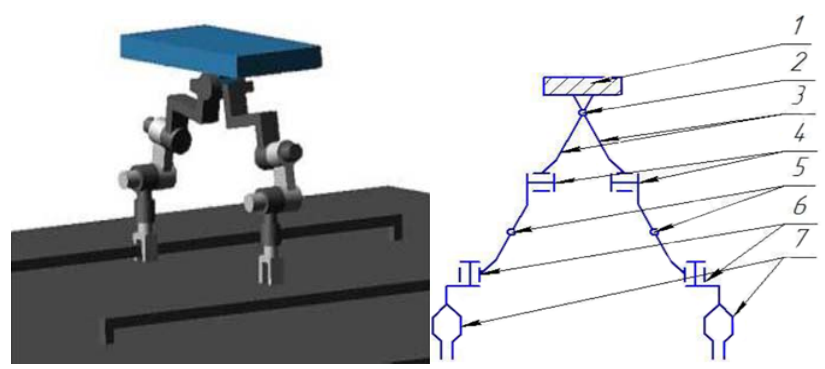

FIGURE I. KINEMATIC STRUCTURE OF TRANSPORT AND MANIPULATION PLATFORM

where: 1- technological platform; 2 - elbow joint; 3 connecting links; 4 - roll joints; 5 - pitch joints; 6 - yaw joints; 7 - grippers

The chosen structure has a kinetic redundancy because of its symmetry, which allows us to operate with both feet equally. At the same time we have the necessary degrees of freedom for positioning and orientating of the gripping. Therefore, such kinematics allows the transport and manipulation system to move on surfaces of any shape with obstacles. The symmetrical design ensures equal opportunities when operating by the right and by the left foot, and allows us to use standardized algorithms for the motion.

As manipulation subsystem we chose the manipulator with a specialized gripper and sex degrees of freedom. Six degrees of freedom is sufficient for accurate positioning of the objects and performance of the necessary functions. To develop approaches to engineering of mechatronic components of the transport and manipulation system we developed its design shape. General view of the multi-functional transport and manipulation is shown in Figure 2.

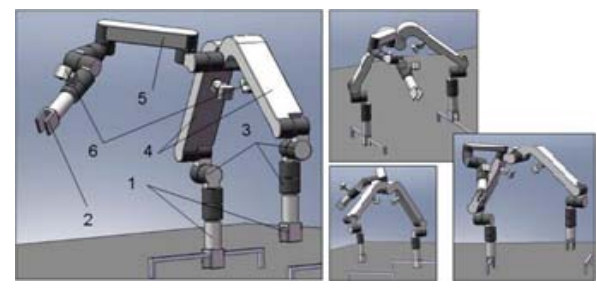

FIGURE II. MULTIFUNCTIONAL TMS 
where: 1 - grippers holding for mounting on the lifting elements of the ISS; 2 - gripping multifunctional; 3 - joints; 4 hardware unit; 5 - arm; 6 - a set of television equipment.

The modular structure of the TMS allows, if necessary, to make the transformation or reconfiguration of the robot to perform the tasks of different groups [5].

\section{SimUlation}

To perform various calculations we constructed threedimensional solid-state computer model of the TMS. The simulation was performed in the MSC.ADAMS package. The study was carried out by performing model simulations of motion and analysis of simulation results.

Simulations have shown the ability of the TMS with the selected kinematics perform the necessary tasks, in particular:

- To move on the rail of the ISS RS, considering the irregularity of their arrangement, the curvature of the surface and the presence of the mating surfaces of the ISS RS; $1.5 \mathrm{~m}$;

- To overcome the gaps in the tracks of the handrails up to

- To carry out inspection operations at the maximum distance to the test object $2.6 \mathrm{~m}$ minimum;

- To perform manipulation operations with a maximum distance to the test object $1.6 \mathrm{~m}$ minimum.

The developed TMS can be located on one handrail on different identically oriented handrails and in various differently orientated handrails (e. g, perpendicular). Therefore, an important expectation was to evaluate the elastic deformation of the corners of aluminum handrails of the ISS $\mathrm{RS}$, which will have an impact on the value of the deflection angle of the TMS (Figure 3).

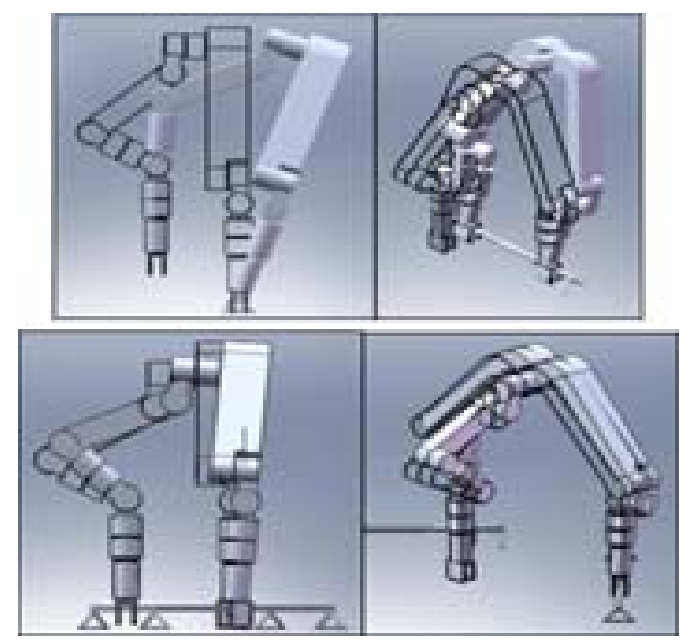

FIGURE III. MOUNTING OF THE TMC ON ONE HANDRAIL (TOP) AND TWO PERPENDICULAR HANDRAILS (BOTTOM)

As the gripper of the TMS we propose the use of one of the two variants of the fixing grippers shown in Figure 4. We prefer the second option (right), equipped with a sixcomponent force and torque sensor; this gripper is shown in the general model of the TMS [6-8].
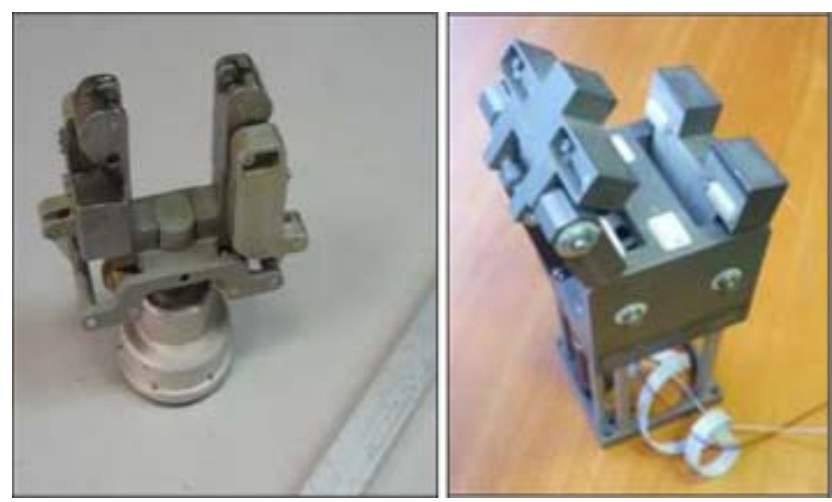

FIGURE IV. VARIANTS OF FIXING GRIPPER

\section{COORDINATED CONTROL OF MANIPULATORS}

For the stable movement along the rails we need to harmonize the movement the two transport manipulators so that:

- They were not released at the same time;

- They are not simultaneously restrained;

- Do not interfere with each other.

To solve these problems we used the principles of control drawn from the ideology of the mobile robots control.

This ideology is described in detail in [9, 10]. Let us say here that the authors applied a similar approach to control a group of robots designed to rescue people at oil platforms in case of disaster.

\section{RESUlTS}

On the basis of the calculations the kinematic robot structure was justified and predictive characteristics of TMS were formulated (Table 1).

TABLE I. PROJECTED CHARACTERISTICS TMS

\begin{tabular}{|l|c|}
\hline \multicolumn{1}{|c|}{ Parameter } & Forecast \\
\hline The number of joints, pieces & 13 \\
\hline Power supply voltage, $\mathrm{V}$ & 27 \\
\hline Maximum power consumption, $\mathrm{W}$ & 600 \\
\hline Power consumption in storage mode, $\mathrm{W}$ & 100 \\
\hline The capacity of an independent source of power Wh (Al) & $2400(90)+2400(90)$ \\
\hline The duration of battery life, $\mathrm{h}$ & 8 \\
\hline Dimensions in the folded state, $\mathrm{mm}$ & $500 \times 500 \times 1200$ \\
\hline Bridging the slopes, $\mathrm{m}$ & $\min 1,5$ \\
\hline Reach the working bodies, $\mathrm{m}$ & $\min 2,6$ \\
\hline
\end{tabular}

Currently the Central Research and Development Institute of Robotics and Technical Cybernetics carries out the work on testing a prototype model of specialized manipulation system (SMS). After that the flight model of the SMS will be developed, and later it will be tested on the ISS in real space (Figure 5). 


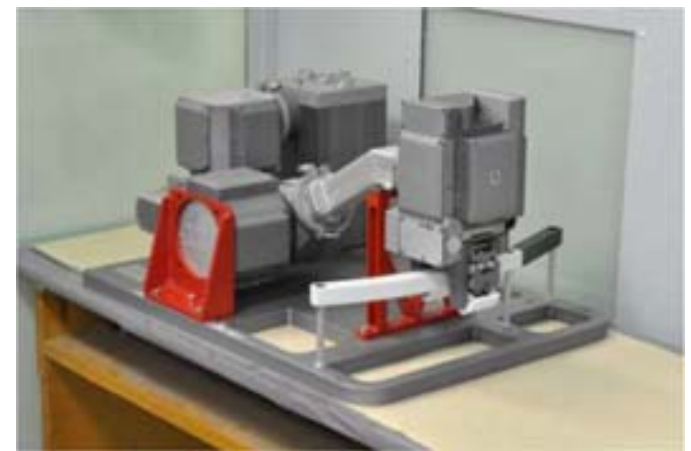

FIGURE V. THE PROTOTYPE OF A SPECIALIZED MANIPULATION SYSTEM

\section{CONCLUSION}

The computer studies have shown:

- Performance and functionality of the TMS basic kinematic scheme;

- Performance of functional modules (joints) of the TMS in outer space, taking into account extreme temperatures;

- The possibility of implementing force-torque-based control on the basis of the studied sensors;

- Functionality of the TMS modules and the potential opportunity for rapid module replacement and reconfiguration.

These given results demonstrate the ability and determine the technical way to create a transport manipulation robotic system to service the outer surface of the spacecraft.

The next step in the development of the TMS is the creation of groups of the organized TMS to perform complex operations on the outer surface of the spacecraft in space. Therefore, in the further work we should pay attention to the development of algorithms and principles of group interaction of the TMS for space. The algorithms of group interactions for terrestrial robotic tools can serve as prototypes. The work on them is carried out in the NNSTU named after R. E. Alekseev, with the support of the Ministry of Education and Science within the frames of the program «Research and development on priority directions of scientific-technological complex of Russia for 2014-2020», the unique identifier of the project: RFMEFI57414X0055.

\section{REFERENCES}

[1] I. A. Kalyaev, A. R. Gaiduk, S. G. Kapustyan. Models and algorithms for collective control in groups of robots. M: FIZMATLIT 2009, p. 280.

[2] 2. S.G. Kapustyan. Methods of self-organization of distributed information and control systems, of intelligent multi-robotic systems // Herald of computer and information technologies. №3 (93), 2012. pp 3541.

[3] Strategy project for development of space activity in Russia up to 2030 and beyond.

[4] Development and research of a method for compensating the dynamic interaction in the system "small spacecraft - manipulator" in the problems of the Earth shooting camera guidance / A. A .Carandaev // Proceedings of the XX International Scientific - Technical Conference "Extreme Robotics. Nano -, micro - and macro-robots(ER - 2009) ". Taganrog: Publishing house TTI SFU, 2009. - P. 98 - 99
[5] A. A. Gradovtsev. Robotic support for future space infrastructure facilities / A. A. Gradovtsev, A. S. Kondratiev A. N. Timofeev // Extreme Robotics: Proceedings of the International scientific and technical Conference November 23-25, 2011, St. Petersburg., 2011.

[6] http://www.lorenz-

messtechnik.de/english/files/publications/Management_Systems_Calibra tion Torque.pdf http://www.lorenzmesstechnik.de/english/company/torque_measurement_technology.php

[7] https://measurementsensors.honeywell.com/ProductDocuments/Manuals /1800/Operating Service/ Manual.pdf

[8] I. A. Vassilyev, S. A. Polovko., E. Y. Smirnova. Organization of group control of mobile robots for special tasks of robotics // Scientific and technical statements STU, № 1 for 2013

[9] I. Vassilyev, A. Koshurina, M. Krasheninnikov, E. Smirnova. The use of mobile robots groups for rescue missions in extreme climatic conditions // Annals of 25th DAAAM International Symposium on Intelligent Manufacturing and Automation, DAAAM 2014 